\title{
Trends in the prevalence of heart diseases over a ten-year period from single-center observations based on
} a large echocardiographic database*

\author{
Hao LU ${ }^{\S 1}$, Wen-zhi PAN ${ }^{\S 1}$, Quan WAN ${ }^{2}$, Lei-lei $\mathrm{CHENG}^{\dagger \star 2}$, Xian-hong SHU ${ }^{2}$, \\ Cui-zhen PAN ${ }^{2}$, Ju-ying QIAN ${ }^{1}$, Jun-bo GE ${ }^{\dagger * 1}$ \\ ( ${ }^{1}$ Department of Cardiology, Zhongshan Hospital, Fudan University, Shanghai Institute of Cardiovascular Diseases, Shanghai 200032, China) \\ ( ${ }^{2}$ Department of Echocardiography, Zhongshan Hospital, Fudan University, Shanghai Institute of Cardiovascular Diseases, Shanghai 200032, China) \\ †E-mail: cheng.leilei@zs-hospital.sh.cn; jbge@zs-hospital.sh.cn \\ Received June 1, 2015; Revision accepted Nov. 7, 2015; Crosschecked Dec. 16, 2015
}

\begin{abstract}
There is a paucity of data regarding trends in the incidence of heart disease in China during recent years. Using a large echocardiography database in our center, we analyzed trends in the prevalence of several common heart diseases from Dec. 2003. This study retrospectively analyzed the echocardiographic database in our Department from 2003 to 2012. A total of 385682 cases were included in the study. The prevalence of rheumatic heart disease decreased over the 10-year period, from $4.04 \%$ in 2003 to $3.06 \%$ in $2012(P<0.01)$. Infective endocarditis also decreased, from a mean prevalence of $0.37 \%$ in July 2003 to $0.27 \%$ in Dec. $2008(P<0.001)$. The prevalence of hypertrophic cardiomyopathy, which includes $20 \%$ apical hypertrophic cardiomyopathy and $20 \%$ hypertrophic obstructive cardiomyopathy, was about $1.8 \%$. The prevalence of the three most common adult congenital heart diseases (CHDs) decreased by about $10 \%$ from July 2003 to Dec. 2008 (all $P<0.001$ ). The prevalence of moderate pulmonary arterial hypertension (PAH) or left ventricular systolic dysfunction (LVSD) decreased during the 10-year period $(P<0.001)$, but there was no change in the prevalence of severe PAH or LVSD $(P>0.05)$. The present study indirectly demonstrates that the prevalence of several common heart diseases in China has declined in recent years.
\end{abstract}

Key words: Prevalence, Trends, Heart diseases, Epidemiology http://dx.doi.org/10.1631/jzus.B1500136

CLC number: R541

\section{Introduction}

From 1990 to 2005, the total number of deaths from cardiovascular disease (CVD) globally increased from 14.4 million to 17.5 million (World Health Organization, 2005). Today, CVD is the largest single contributor to global mortality and will continue to

\footnotetext{
$₫$ Corresponding authors

$\S$ The two authors contributed equally to this work

* Project supported by the National Natural Science Foundation of China (Nos. 81400318 and 81300209)

(i) ORCID: Hao LU, http://orcid.org/0000-0002-0322-4400

(C) Zhejiang University and Springer-Verlag Berlin Heidelberg 2016
}

dominate mortality trends in the future. Notably, over $80 \%$ of deaths from CVD occur in low and middle income countries (World Health Organization, 2009). The global disease burden will likely shift from infectious diseases to chronic diseases within the next few decades.

China has experienced an epidemiological shift from infectious to chronic diseases in a much shorter time than many other countries (Yang et al., 2008). In China, the CVD burden is an important public health issue. The number of Chinese with CVD is estimated at 230 million individuals (NCCD, 2010). Moreover, CVD accounts for $41 \%$ of deaths from all causes and is the leading cause of death in China (He et al., 2005; 
Li et al., 2014). However, data regarding the trends in the prevalence of heart disease in recent years are lacking. Our center, the Shanghai Institute of Cardiovascular Diseases (Shanghai, China), is one of the three largest heart research centers in China. The objective of our study was to analyze trends in the prevalence of several common heart diseases from 2003 to 2012 using a large echocardiographic database at our center.

\section{Materials and methods}

\subsection{Data analysis}

This analysis used the echocardiographic database of outpatients who were referred to our department from 2003 to 2012. For patients who underwent echocardiography (ECG) examination more than once within one year, we used the first result or the final definitive diagnostic result. Only 2-dimensional (2D) transthoracic echocardiograms were used for analysis (2D imaging, M-mode imaging, Doppler imaging). 3D-echocardiograms, tissue Doppler images, and transesophageal echocardiograms were excluded. The echocardiographic diagnosis was based on recommendations of the American Society of Echocardiography (Zoghbi et al., 2003; Douglas et al., 2011). Patients younger than 18 years were excluded. The prevalence of a disease was calculated as the ratio of the number of patients with the disease to the total annual number of patients. The study followed the principles of the Declaration of Helsinki and was approved by the Institutional Review Board of Zhongshan Hospital, Fudan University (Shanghai, China).

\subsection{Diseases for analysis}

\subsubsection{Valvular heart diseases (VHDs)}

VHDs were classified into rheumatic heart disease (RHD), non-rheumatic VHD, and post valve surgery. We analyzed only aortic and mitral valve abnormalities; tricuspid and pulmonary valve abnormalities were not included in the analysis. Minor valve regurgitation was not regarded as VHD because it may occur in many healthy patients, but minor valve stenosis was included. Infectious endocarditis was also included as a type of VHD.

\subsubsection{Cardiomyopathy}

Cardiomyopathy included hypertrophic cardiomyopathy (HCM), non-compaction of the ventricular myocardium, arrhythmogenic right ventricular cardiomyopathy, and restrictive cardiomyopathy. Ventricular cardiomyopathy and restrictive cardiomyopathy were initially diagnosed by ECG, but the final diagnosis of the disease was made by reviewing the medical records and other examinations. HCM was classified into three types: apical HCM, hypertrophic obstructive cardiomyopathy (HOCM), and hypertrophic nonobstructive cardiomyopathy (HNOCM). Dilated cardiomyopathy was not included in the analysis because its diagnosis cannot be based solely on ECG.

\subsubsection{Congenital heart disease (CHD)}

We analyzed four of the most common CHDs: atrial septal defect (ASD), ventricular septal defect (VSD), patent ductus arteriosus (PDA), and bicuspid aortic valve (BAV).

2.2.4 Pulmonary arterial hypertension (PAH) and left ventricular systolic dysfunction (LVSD)

We enrolled patients with moderate PAH (pulmonary artery systolic pressure (PASP), $50-69 \mathrm{mmHg}$ ) or severe PAH (PASP, $\geq 70 \mathrm{mmHg}$ ), where PASP was calculated as the sum of the tricuspid jet gradient. Also, PAH was divided into CHD associated PAH and other types of PAH. LVSD patients had a left ventricular ejection fraction between $30 \%$ and $45 \%$ (moderate) or $<30 \%$ (severe).

\subsection{Statistical analysis}

Continuous variables are presented as the mean \pm standard deviation (SD) and categorical variables as percentages. The prevalence of diseases was compared using the $\chi^{2}$ test. $P<0.05$ was considered to represent statistical significance. The relative change in prevalence of disease between the first and second 5 -year periods was calculated as follows: (prevalence in Dec. 2008-prevalence in July 2003)/prevalence in July $2003 \times 100 \%$. We defined a change in relative value greater than $10 \%$ as a marked change, while a difference of less than $10 \%$ was regarded as not significant. Statistical analyses were performed using the SPSS 13.0 software package (SPSS Inc., Chicago, Illinois, USA). 


\section{Results}

A total of 385682 cases were included in the study. Demographic characteristics of patients are given in Table 1. The proportion of male patients was about $50 \%$ each year, ranging from $49.12 \%$ to $52.32 \%$.
The mean age was $(54.97 \pm 17.35)$ years. The proportion of patients undergoing ECG remained at $15 \%$ each year, while outpatient visits increased every year, from 80856 in 2003 to 423561 in 2012 . The trends in the prevalence of heart diseases during the 10-year period are shown in Table 2.

Table 1 Demographic characteristics of outpatients undergoing transthoracic echocardiogram examination in our department from 2003 to 2012

\begin{tabular}{|c|c|c|c|c|c|}
\hline Year & Number of case & Male $(\%)$ & Age (year) & $\begin{array}{l}\text { Total number of outpatient visits } \\
\text { (person-time) }\end{array}$ & $\begin{array}{c}\text { Percentage of outpatient } \\
\text { undergoing ECG }(\%)\end{array}$ \\
\hline 2003 & 11086 & 51.19 & $49.38 \pm 17.88$ & 80856 & 13.71 \\
\hline 2004 & 14656 & 52.32 & $54.39 \pm 16.06$ & 103526 & 14.16 \\
\hline 2005 & 23655 & 51.00 & $54.28 \pm 16.06$ & 153420 & 15.42 \\
\hline 2006 & 28210 & 50.54 & $54.81 \pm 16.36$ & 184755 & 15.27 \\
\hline 2007 & 36926 & 49.12 & $55.21 \pm 16.19$ & 244555 & 15.10 \\
\hline 2008 & 46414 & 49.94 & $55.47 \pm 18.61$ & 293538 & 15.81 \\
\hline 2009 & 53145 & 49.49 & $55.76 \pm 16.10$ & 334517 & 15.89 \\
\hline 2010 & 54247 & 51.42 & $55.87 \pm 15.84$ & 375271 & 14.45 \\
\hline 2011 & 55597 & 50.56 & $57.08 \pm 15.69$ & 394918 & 14.08 \\
\hline 2012 & 61746 & 51.80 & $57.10 \pm 16.50$ & 423561 & 14.57 \\
\hline
\end{tabular}

Table 2 Trends in the prevalence of several common heart diseases in China from 2003 to 2012

\begin{tabular}{|c|c|c|c|c|c|c|c|c|c|c|c|c|c|c|}
\hline \multirow[b]{2}{*}{ Disease } & \multicolumn{12}{|c|}{ Prevalence of several common heart diseases $(\%)$} & \multirow{2}{*}{$\begin{array}{c}\text { Change }{ }^{*} \\
(\%)\end{array}$} & \multirow[b]{2}{*}{$P^{* *}$} \\
\hline & 2003 & 2004 & 2005 & 2006 & 2007 & 2008 & 2009 & 2010 & 2011 & 2012 & $\begin{array}{c}\text { First } \\
5 \text { years }\end{array}$ & $\begin{array}{c}\text { Last } \\
5 \text { years }\end{array}$ & & \\
\hline RHD & 4.04 & 5.49 & 3.73 & 3.71 & 3.43 & 3.11 & 2.91 & 3.10 & 3.05 & 3.06 & 3.87 & 3.04 & -21.47 & $<0.001$ \\
\hline IE & 0.47 & 0.48 & 0.27 & 0.41 & 0.31 & 0.27 & 0.20 & 0.29 & 0.30 & 0.31 & 0.35 & 0.26 & -25.56 & $<0.001$ \\
\hline Post valve surgery & 5.16 & 5.04 & 4.72 & 3.38 & 4.11 & 4.32 & 4.38 & 4.36 & 4.62 & 4.40 & 4.18 & 4.42 & 5.79 & 0.002 \\
\hline Non-RHD VHD & 4.66 & 5.15 & 5.01 & 4.27 & 4.27 & 4.33 & 3.82 & 4.64 & 4.54 & 4.65 & 4.67 & 4.33 & -7.68 & 0.006 \\
\hline HOCM & 0.32 & 0.42 & 0.37 & 0.37 & 0.33 & 0.29 & 0.37 & 0.42 & 0.48 & 0.45 & 0.37 & 0.40 & 8.41 & 0.008 \\
\hline HNOCM & 0.89 & 1.35 & 1.08 & 1.23 & 0.95 & 0.94 & 0.95 & 1.11 & 1.15 & 0.99 & 1.11 & 1.04 & -6.31 & 0.064 \\
\hline ApHCM & 0.39 & 0.33 & 0.34 & 0.32 & 0.25 & 0.16 & 0.29 & 0.52 & 0.33 & 0.69 & 0.30 & 0.33 & 10.01 & 0.202 \\
\hline Total of HCM & 1.60 & 2.10 & 1.79 & 1.92 & 1.53 & 1.39 & 1.61 & 2.05 & 2.17 & 2.14 & 1.78 & 1.76 & -1.12 & 0.784 \\
\hline ARVD & 0.00 & 0.00 & 0.00 & 0.00 & 0.01 & 0.01 & 0.00 & 0.01 & 0.01 & 0.02 & 0.00 & 0.01 & 67.96 & 0.370 \\
\hline $\mathrm{RCM}$ & 0.00 & 0.01 & 0.02 & 0.01 & 0.01 & 0.02 & 0.02 & 0.05 & 0.03 & 0.06 & 0.01 & 0.03 & 216.17 & $<0.001$ \\
\hline NCVM & 0.02 & 0.05 & 0.02 & 0.04 & 0.03 & 0.02 & 0.05 & 0.08 & 0.06 & 0.04 & 0.04 & 0.05 & 36.83 & 0.016 \\
\hline ASD & 3.10 & 2.89 & 2.42 & 2.34 & 2.20 & 2.23 & 2.26 & 2.03 & 2.19 & 2.34 & 2.38 & 2.27 & -5.90 & $<0.001$ \\
\hline VSD & 2.11 & 1.88 & 1.40 & 1.47 & 1.27 & 1.45 & 1.28 & 1.15 & 1.12 & 1.21 & 1.44 & 1.24 & -13.73 & $<0.001$ \\
\hline PDA & 0.78 & 0.76 & 0.59 & 0.57 & 0.53 & 0.53 & 0.52 & 0.55 & 0.48 & 0.54 & 0.59 & 0.52 & -11.52 & 0.017 \\
\hline $\mathrm{ASD}+\mathrm{VSD}+\mathrm{PDA}$ & 5.99 & 5.53 & 4.40 & 4.39 & 4.00 & 4.21 & 4.07 & 4.09 & 3.80 & 4.09 & 4.41 & 3.94 & -10.82 & $<0.001$ \\
\hline BAV & 0.65 & 0.85 & 0.61 & 0.74 & 0.55 & 0.62 & 0.74 & 0.79 & 0.90 & 1.08 & 0.65 & 0.72 & 9.24 & 0.058 \\
\hline CHD associated PAH & 1.71 & 1.30 & 1.14 & 1.22 & 1.02 & 0.91 & 0.85 & 0.82 & 0.83 & 0.97 & 1.14 & 0.85 & -25.65 & $<0.001$ \\
\hline Other types of PAH & 7.73 & 9.40 & 5.50 & 7.58 & 6.82 & 5.70 & 3.64 & 6.22 & 5.98 & 6.06 & 7.09 & 5.39 & -24.01 & $<0.001$ \\
\hline Moderate PAH & 7.48 & 8.54 & 6.64 & 7.09 & 6.18 & 5.09 & 4.79 & 5.21 & 5.01 & 5.31 & 6.87 & 5.02 & -26.87 & $<0.001$ \\
\hline Severe PAH & 1.96 & 2.16 & 1.53 & 1.71 & 1.66 & 1.52 & 1.59 & 1.83 & 1.81 & 1.72 & 1.72 & 1.69 & -1.37 & 0.638 \\
\hline Moderate LVSD & 4.26 & 4.23 & 4.27 & 4.19 & 3.44 & 3.22 & 3.34 & 3.33 & 3.64 & 3.12 & 3.94 & 3.39 & -14.20 & $<0.001$ \\
\hline Severe LVSD & 0.92 & 0.96 & 0.96 & 0.76 & 0.66 & 0.61 & 0.91 & 0.89 & 0.90 & 1.17 & 0.79 & 0.84 & 5.04 & 0.256 \\
\hline
\end{tabular}

RHD, rheumatic heart disease; IE, infective endocarditis; VHD, valvular heart disease; HOMC, hypertrophic obstructive cardiomyopathy; HNOMC, hypertrophic non-obstructive cardiomyopathy; ApHCM, apical hypertrophic cardiomyopathy; HCM, hypertrophic cardiomyopathy; ARVD, arrhythmogenic right ventricular cardiomyopathy; RCM, restrictive cardiomyopathy; NCVM, non-compaction of ventricular myocardium hypertrophic; ASD, atrial septal defect; VSD, ventricular septal defect; PDA, patent ductus arteriosus; BAV, bicuspid aortic valve; CHD, congenital heart disease; PAH, pulmonary arterial hypertension; LVSD, left ventricular systolic dysfunction. ${ }^{*}$ The relative change value of prevalence of disease between the first 5 years and last 5 years. ${ }^{* *}$ Statistics values for comparisons of prevalence of disease between the first 5 years and last 5 years 


\subsection{VHD}

There was a trend towards decreasing prevalence of RHD over the 10-year period, from $4.04 \%$ in 2003 to $3.06 \%$ in $2012(P<0.01)$. Infective endocarditis also decreased, from an average prevalence of $0.37 \%$ in July 2003 to $0.27 \%$ in Dec. $2008(P<0.001)$. There was no clear trend in the percentage of patients with non-rheumatic VHD (ranging from $3.82 \%$ to $5.15 \%$ ) and patients having undergone valve surgery (ranging from $3.38 \%$ to $5.16 \%$ ).

\subsection{Cardiomyopathy}

The prevalence of HCM, which included $20 \%$ apical HCM and 20\% HOCM, was about 1.8\%. There was no obvious change in the prevalence of HCM or its subgroups over the 10-year period. Other types of cardiomyopathy, including arrhythmogenic right ventricular cardiomyopathy, restrictive cardiomyopathy, and non-compaction of ventricular myocardium, were rather rare $(0-0.08 \%)$.

\subsection{CHD}

The prevalence of the three most common CHDs, ASD, VSD, and PDA, decreased by about $10 \%$ from the first to the second five-year period (all $P<0.001$, Table 2); the prevalence of another common CHD, $\mathrm{BAV}$, did not change markedly (about $0.7 \%$ ).

\subsection{PAH and LVSD}

The prevalence of CHD-associated PAH (or other types of $\mathrm{PAH}$ ) and moderate $\mathrm{PAH}$ decreased during the 10-year period $(P<0.001)$, while that of severe PAH did not change significantly (1.74\% in July 2003 , $1.71 \%$ in Dec. 2008; $P=0.697)$. Similarly, the average prevalence of patients with moderate LVSD declined over the 10-year period, from $4.26 \%$ in 2003 to $3.12 \%$ in $2012(P<0.001)$, while that of severe LVSD remained unchanged $(0.80 \%$ in July $2003,0.85 \%$ in Dec. 2008; $P=0.261)$.

\section{Discussion}

The Department of Cardiology, Shanghai Institute of Cardiovascular Diseases (Shanghai, China), which treats patients from all over the nation, is one of the three largest and most recognized heart research centers in China. There are more than 10000 inpatient and 300000 outpatient visits per year. Thus, the patients and their respective data reasonably reflect the trends in the prevalence of heart disease within the country as a whole. A total of 385682 cases were included in this study. The main findings were as follows: (1) the prevalence of RHD decreased over the 10 -year period, from $4.04 \%$ in 2003 to $3.06 \%$ in 2012; (2) HCM was not rare and apical HCM was common $(20 \%)$; however, other types of cardiomyopathy were rather uncommon; (3) When comparing the average prevalence of the first 5-year period with that of the last 5-year period, the prevalence of the three most common adult CHDs decreased by about $10 \%$; (4) the prevalence of patients with moderate PAH or LVSD decreased during the 10-year period, while there was no change in the prevalence of severe PAH or LVSD.

RHD remains a significant health burden in China. The prevalence of chronic RHD is estimated to be 186/100 000 adults, which is 10 times higher than that in industrialized countries, such as Canada and Japan (Zhimin et al., 2006). The prevalence of acute rheumatic fever and RHD has been declining since the 1950s (Chen et al., 2003). Our study showed that RHD accounted for 3\%-5\% of outpatient visits; furthermore, the prevalence of RHD had continued to decline over the 10-year period of the study. Infective endocarditis also decreased, but the percentage of patients with non-rheumatic VHD and of patients having undergone valve surgery did not change significantly.

HCM is a global disease and is present in about 2 of every 1000 young adults in the USA (Maron et al., 1995). In China, HCM is not so rare; the estimated prevalence is about 80 per 100000 adults (Zou et al., 2004). Apical HCM was first described in Japan (Sakamoto et al., 1976), where it accounts for $13 \%$ to $25 \%$ of the entire HCM population (Eriksson et al., 2002). In western countries it is less common, accounting for $3 \%$ to $11 \%$ of all HCM patients (Moro et al., 1995; Eriksson et al., 2002). Apical HCM was present in about $20 \%$ of the entire HCM population in our study, which is similar to the incidence in the Japanese population. Generally, HOCM is found in about $25 \%$ of patients with HCM (Wigle et al., 1985). In the present study, $20 \%$ of HCM was HOCM.

The overall incidence of CHD is 8.2/1000 total births in China (Yang et al., 2009), which is similar to 
that of developed countries (Mitchell et al., 1971). The most common types of CHD in newborns are VSD (34.0\%), PDA (23.7\%), and ASD (10.8\%) (Mitchell et al., 1971). Data on the size and composition of the adult CHD population are lacking, but can be assumed to be ever-changing. It is estimated that there are about 2.8 adults with CHD per 1000 of the population (Warnes et al., 2001). In the present study, adult CHD was present in $5 \%$ of patients; the most common CHDs were ASD, VSD, BAV, and PDA. The prevalence of the three most common CHDs (ASD, VSD, and PDA) decreased over the 10 -year period. There are two possible explanations for this phenomenon: first, patients with CHD are diagnosed and treated at a younger age due to the increasing availability and use of improved examination techniques; second, the incidence of live-born CHD has been significantly reduced through fetal ECG (Yang et al., 2009).

PAH was present in about $7 \%-9 \%$ of all patients, while LVSD was present in about $4 \%-5 \%$. The prevalence of CHD associated with PAH decreased over the 10-year period. This may be due to the decline in the incidence of adult CHD and early treatment of this disease. Moderate PAH decreased, while severe PAH did not change. Similarly, the prevalence of patients with moderate LVSD decreased over the 10 -year period, but there was no change in the prevalence of severe LVSD. There remains a lack of effective methods for treatment of severe PAH or LVSD. Further efforts should be made to prevent cardiovascular diseases from developing into severe PAH or severe LVSD.

\section{Limitations}

There were several limitations of the study. First, this is a single center study and the results of the study may only broadly reflect the trends in the prevalence of heart diseases in China. Second, the epidemiological analysis was based on a patient population referred to our department; this is not a community population. Thus, the prevalence of heart diseases was not their true epidemiological prevalence. The prevalence of heart diseases in the present study only indirectly reflects the prevalence of heart diseases in the general population. Third, the diagnosis of heart diseases included in the study was based on ECG.
Therefore, many other common diseases that could be diagnosed by clinical examination or other methods, such as arrhythmia, dilated cardiomyopathy, or coronary heart disease, were not analyzed in the present study.

In conclusion, our study demonstrates a decreasing trend in the prevalence of RHD and infective endocarditis from 2003 to 2012 . When comparing the prevalence in the first 5 -year period with that of the second, the prevalence of the three most common CHDs (ASD, VSD, and PDA) had decreased. This study indirectly demonstrated the trends in the prevalence of several common heart diseases in China from 2003 to 2012.

\section{Compliance with ethics guidelines}

Hao LU, Wen-zhi PAN, Quan WAN, Lei-lei CHENG, Xian-hong SHU, Cui-zhen PAN, Ju-ying QIAN, and Jun-bo GE declare that they have no conflict of interest.

All procedures followed were in accordance with the ethical standards of the responsible committee on human experimentation (institutional and national) and with the Helsinki Declaration of 1975, as revised in 2008 (5).

\section{References}

Douglas, P.S., Garcia, M.J., Haines, D.E., et al., 2011. Appropriate use criteria for echocardiography. J. Am. Soc. Echocardiogr., 24(3):229-267. http://dx.doi.org/10.1016/j.echo.2010.12.008

Chen, H.Z., Fan, W.H., Jin, X.J., et al., 2003. Changing trends of etiologic characteristics of cardiovascular diseases among inpatients in Shanghai: a retrospective observational study from 1948 to 1999. Chin. J. Intern. Med., 42(12):829-832 (in Chinese).

Eriksson, M.J., Sonnenberg, B., Woo, A., et al., 2002. Long-term outcome in patients with apical hypertrophic cardiomyopathy. J. Am. Coll. Cardiol., 39(4):638-645. http://dx.doi.org/10.1016/S0735-1097(01)01778-8

He, J., Gu, D., Wu, X., et al., 2005. Major causes of death among men and women in China. N. Engl. J. Med., 353(11):1124-1134. http://dx.doi.org/10.1056/NEJMsa050467

Li, J., Li, X., Wang, Q., et al., 2014. ST-segment elevation myocardial infarction in China from 2001 to 2011 (the China PEACE-Retrospective Acute Myocardial Infarction Study): a retrospective analysis of hospital data. Lancet, 385(9966):441-451. http://dx.doi.org/10.1016/S0140-6736(14)60921-1

Maron, B.J., Gardin, J.M., Flack, J.M., et al., 1995. Prevalence of hypertrophic cardiomyopathy in a general population of young adults: echocardiographic analysis of 4111 subjects in the CARDIA Study. Circulation, 92(4):785-789. http://dx.doi.org/10.1161/01.CIR.92.4.785

Mitchell, S.C., Korones, S.B., Berendes, H.W., 1971. Congenital 
heart disease in 56,109 births. Incidence and natural history. Circulation, 43(3):323-332.

http://dx.doi.org/10.1161/01.CIR.43.3.323

Moro, E., D'Angelo, G., Nicolosi, G.L., et al., 1995. Long-term evaluation of patients with apical hypertrophic cardiomyopathy. Correlation between quantitative echocardiographic assessment of apical hypertrophy and clinicalelectrocardiographic findings. Eur. Heart J., 16(2):210-217.

NCCD (National Center for Cardiovascular Disease of China), 2010. Report on Cardiovascular Disease in China (20082009). Encyclopedia of China Publishing House, p.7 (in Chinese).

Sakamoto, T., Tei, C., Murayama, M., et al., 1976. Giant T wave inversion as a manifestation of asymmetrical apical hypertrophy $(\mathrm{AAH})$ of the left ventricle: echocardiographic and ultrasono-cardiotomographic study. Jpn. Heart J., 17(5):611-629. http://dx.doi.org/10.1536/ihj.17.611

Warnes, C.A., Liberthson, R., Danielson, G.K., et al., 2001. Task force 1: the changing profile of congenital heart disease in adult life. J. Am. Coll. Cardiol., 37(5): 1170-1175. http://dx.doi.org/10.1016/S0735-1097(01)01272-4

Wigle, E.D., Sasson, Z., Henderson, M.A., et al., 1985. Hypertrophic cardiomyopathy: the importance of the site and extent of hypertrophy: a review. Prog. Cardiovasc. Dis., 28(1):1-83. http://dx.doi.org/10.1016/0033-0620(85)90024-6

World Health Organization, 2005. Preventing chronic diseases: a vital investment. Available from http://www.who.int/ chp/chronic_disease_report/full_report.pdf [Accessed on Apr. 10, 2015].

World Health Organization, 2009. World health statistics 2009. Geneva: World Health Organization. Available from http://www.who.int/whosis/whostat/EN_WHS09_Full.pdf [Accessed on Apr. 10, 2015].

Yang, G., Kong, L., Zhao, W., et al., 2008. Emergence of chronic non-communicable diseases in China. Lancet, 372(9650):1697-1705.

http://dx.doi.org/10.1016/S0140-6736(08)61366-5

Yang, X.Y., Li, X.F., Lü, X.D., et al., 2009. Incidence of congenital heart disease in Beijing, China. Chin. Med. J. (Engl.), 122(10):1128-11132.

Wang, Z.M., Zou, Y.B., Song, L., et al., 2006. Prevalence of chronic rheumatic heart disease in Chinese adults. Int. J. Cardiol., 107(3):356-359.

http://dx.doi.org/10.1016/j.ijcard.2005.03.048

Zoghbi, W.A., Enriquez-Sarano, M., Foster, E., et al., 2003. Recommendations for evaluation of the severity of native valvular regurgitation with two-dimensional and Doppler echocardiography. J. Am. Soc. Echocardiogr., 16(7): 777-802. http://dx.doi.org/10.1016/S0894-7317(03)00335-3

Zou, Y., Song, L., Wang, Z., et al., 2004. Prevalence of idiopathic hypertrophic cardiomyopathy in China: a population-based echocardiographic analysis of 8080 adults. Am. J. Med., 116(1):14-18.

http://dx.doi.org/10.1016/j.amjmed.2003.05.009

\section{中文概要}

题 目: 从单中心大型超声心动图数据分析近十年中国 心脏疾病患病率的变化趋势

目 的: 中国心脏疾病近年患病率趋势变化的相关研究较 少, 本研究利用复旦大学附属中山医院心血管病 研究所中心大型的超声心动图数据, 观察 2003 年至 2012 年心脏疾病患病率的变化。

创新点: 本研究间接地反映近十年来我国心脏疾病患病率 趋势变化的情况。

方 法: 回顾性地分析了本中心 2003 年至 2012 年的超声 心动图数据, 共纳入了 385682 名患者。

结 论: 从 2003 年至 2012 年, 风湿性心脏病和感染性心 内膜炎的患者率明显下降; 2003-2007 年期间包 括房间隔缺损、室间隔缺损和动脉导管未闭的先 天性心脏病的患病率较 2008-2012 年明显下降; 重度肺动脉高压、重度左室收缩功能不全和肥厚 型心肌病的患病率在十年间没有明显变化。

关键词: 患病率; 趋势; 心脏疾病; 流行病学 